\title{
Isometric Thumb Exertion Induces B Cell and T Cell Lymphocytosis in Trained and Untrained Males: Physical Aptitude Determines Response Profiles
}

\author{
Adam Michael Szlezak (Corresponding author) \\ Division of Sports Science, School of Allied Health Sciences, \\ Griffith University, Parklands Dr, Southport QLD 4215, Australia \\ E-mail: adam.szlezak@griffithuni.edu.au \\ Lotti Tajouri \\ Faculty of Health Science \& Medicine, Bond University, Gold Coast QLD 4229, Australia \\ E-mail: 1tajouri@bond.edu.au \\ James Keane \\ Faculty of Health Science \& Medicine, Bond University, Gold Coast QLD 4229, Australia \\ E-mail: keanejames4@gmail.com \\ Siri Lauluten Szlezak \\ Faculty of Health Science \& Medicine, Bond University, \\ Gold Coast QLD 4229, Australia \\ E-mail: siri.szlezak@gmail.com \\ Clare Minahan \\ Division of Sports Science, School of Allied Health Sciences, \\ Griffith University, Parklands Dr, Southport QLD 4215, Australia \\ E-mail: c.minahan@griffith.edu.au
}

Received: 23-11- 2015

doi:10.7575/aiac.ijkss.v.4n.1p.55
Accepted: 25-01- 2016

Published: 31-01- 2016

\begin{abstract}
Purpose: The present study examined the effect of low-dose thumb exertion on lymphocyte subpopulation trafficking. The potential role of blood lactate in mediating lymphocyte redistribution was also investigated. Methods: 27 male participants (18 weightlifting-trained; 9 untrained) were separated into 3 groups of 9 (Weightlifting and Untrained Experimental: WLEXP, UTEXP; Weightlifting Placebo: WLPLA). WLEXP and $\mathrm{UT}_{\text {EXP }}$ performed 4x60 second isometric thumb intervals separated by 60 second rest intervals in a single-blinded placebo-controlled study. Participants were assessed over a 60 minute post-intervention recovery period for pain, blood lactate and lymphocyte subpopulation counts. Results: WLPLA did not change for any measured variable $(p>0.05)$. The two experimental groups increased significantly $(\mathrm{p}<0.01)$ in thumb pain post-intervention (WLEXP:4.92/10; $\mathrm{UT}_{\mathrm{EXP}}$ :2.92/10) however only WLEXP remained elevated across all time-points. Blood lactate increased for both experimental groups post-intervention $(p<0.01)$ whilst peak concentrations (UT EXP: $2.2 \mathrm{mmol} / \mathrm{L}$; WLEXP: $2.4 \mathrm{mmol} / \mathrm{L}$ ) and temporal profiles were not different between groups ( $p>0.05$ ). No differences in cell count were seen for CD56+/CD16+ lymphocytes across time for any group ( $\mathrm{p}>0.05$ ). $\mathrm{UT}_{\mathrm{EXP}}$ showed an early significant increase (20 min post-intervention) in CD4+CD3+ $(20.78 \%, \mathrm{p}<0.01), \mathrm{CD} 8+\mathrm{CD} 3+$ $(15.25 \%, \mathrm{p}<0.01)$ and $\mathrm{CD} 19+(18.11 \%, \mathrm{p}=0.013)$ cell count before returning to levels not different from baseline by the final time-point $(\mathrm{p}>0.05)$. Conversely, WLEXP group showed no early increase followed by a delayed increase in cell count evident at the final time-point; CD4+CD3+ (19.06\%, p<0.01), CD8+CD3+ (11.46\%, p=0.033) and CD19+ $(28.87 \%, \mathrm{p}<0.01)$. Blood lactate was not correlated with lymphocyte counts. Conclusions: Physical aptitude and not cellular energy demand influences the lymphocyte response to resistance-exercise.
\end{abstract}

Keywords: B-Lymphocytes; Exercise; Lactic Acid; Lymphocytosis; Resistance Training; T-Lymphocytes

\section{Introduction}

Resistance-exercise is recognised as a key component of contemporary athletic training (Azeem \& Kumar, 2011; Ranisavljev \& Vladimir, 2010) and as such, the biological consequences of this exercise mode require thorough investigation. Considering the central role of the immune system in both host-defence and physiological adaptation (Luckheeram, Zhou, Verma, \& Xia, 2012; Mosser \& Edwards, 2008; Sonnet et al., 2006), a clinically relevant 
understanding of the interaction between the immune system and resistance-exercise is of great importance for exercise professionals. Before this can occur however, exercise-induced leukocyte responses and the mechanisms through which these responses transpire must be characterised.

Based on preliminary research, it is suggested that leukocyte responses to exercise are not random, but occur for specific functional purposes (Freidenreich \& Volek, 2012; Walsh et al., 2011). Research is therefore warranted which investigates the relationship between resistance-exercise and those particular leukocytes involved in highly organised and regulated roles. Pleitrophic cells such as the lymphocytes are one important example, since they orchestrate fundamental biological tasks including cell-mediated and humoral immunity, coordination of innate immune responses and in regards to exercise, facilitate physiological adaption such as tissue remodelling (LeBien \& Tedder, 2008; Luckheeram et al., 2012). Currently, the clinically relevant implications of exercise-induced changes to leukocyte homeostasis (distribution, proliferation and function) are unknown (Dohi et al., 2001; Mayhew, Thyfault, \& Koch, 2005; Walsh et al., 2011). Additionally, there is a complete gap in the literature regarding potential immune responses to low-dose exercise (Walsh et al., 2011). Recent research has suggested that exercise intensity and duration are linked to leukocyte redistribution (Bush et al., 1999; Kraemer et al., 1999), however lymphocytes may respond through a different mechanism. Preliminary research in females has shown (Miles et al., 2003) that the greatest lymphocyte responses to resistance-exercise were associated with the highest post-exercise blood lactate levels; that is, high lactate responders $(\geq 11.92 \mathrm{mmol} / \mathrm{L})$ showed significantly greater lymphocyte count elevations than lower lactate responders $(\leq 7.62 \mathrm{mmol} / \mathrm{L})$. Furthermore, this response was not observed for monocytes and or granulocytes suggesting that cellular energy demand may selectively govern the lymphocytic response to resistance-exercise. Insight into the mechanisms through which resistance-exercise affects lymphocyte homeostasis is a key milestone in determining the functional consequences of this exercise mode, and thus warrants further investigation. Although blood lactate concentration cannot determine the exact rate or volume of energy produced anaerobically, it can be used to demonstrate a rise in cellular energy demand and anaerobic energy expenditure (Fernandez, Mendez-Villanueva, \& Pluim, 2006; Spurway, 1992; Wirtz, Wahl, Kleinöder, \& Mester, 2014). From a practical perspective, knowledge that blood lactate is associated with selective regulation of lymphocyte trafficking would influence the way in which anaerobic physical activity (e.g. resistance-training; Wirtz et al., 2014) is prescribed.

A significant challenge with investigating the relationship between lymphocytes and resistance-exercise is ensuring that the exercise intervention can be tightly controlled. A caveat with the existing literature is that it is dominated by largedose exercise protocols encompassing multiple skeletal muscles and joints (Freidenreich \& Volek, 2012). Due to potential inter-individual differences in biomechanics such as joint stability (Solomonow \& Krogsgaard, 2001) and motor control (Frank, Kobesova, \& Kolar, 2013), a researcher's ability to standardize performance of an exercise intervention is adversely affected when employing large dose, multiple-joint protocols. Since the link between blood lactate and lymphocyte redistribution was observed through a large exercise-dose (squat) protocol with previously untrained-female subjects (Miles et al., 2003), the present study aims to employ a more controllable exerciseintervention, i.e. an isometric thumb manoeuvre (Punsola-Izard, Salas-Gómez, Sirvent-Rivalda, \& Esquirol-Caussà, 2012), and a male population. This approach will facilitate reliable examination of lymphocyte responses to resistanceexercise and associated blood lactate changes whilst producing findings relevant to males. Additionally, subjects of differing physical aptitude should be studied to identify if blood lactate or other variables influence lymphocyte-specific trafficking post-exercise. Finally, the study of lymphocyte responses to an isometric thumb exercise protocol will provide novel insight into the potential effect of low-dose exercise on the immune system. In the present study, the following hypotheses were made: i) Short duration isometric thumb exertion will mobilise lymphocyte subpopulations and increase blood lactate concentration; ii) An increase in circulating lymphocyte count is related to an increase in blood lactate concentration; iii) Lymphocyte responses will vary between experimental groups and this will be explained by variances in blood lactate responses.

\section{Methods}

\subsection{Participants}

Prior to recruitment of subjects, this study received full ethical approval from the Griffith University Human Research Ethics Committee. The study conformed to standards set out in the Declaration of Helsinki and its later amendments or comparable ethical standards. Before being admitted to the study, all participants were given a detailed explanation of the study procedures, experimental interventions and potential risks involved. Each participant then signed a written consent form. Twenty seven male subjects (18-40 yr) voluntarily participated in the present study and all twenty seven completed the study. Eighteen men were considered weightlifting athletes who ranged from local to state-level competitors and trained at a frequency of 2 or more weightlifting sessions per week (Table 1). The other nine men were untrained (not participating in any regular exercise and had not performed resistance-exercise for at least 12 months). The untrained subjects were grouped together into the Untrained experimental group (UT $\mathrm{EXP}_{\mathrm{P}}$ n=9). Weightlifting athletes were randomly allocated into one of two groups (using the concealed, third party randomization method described by Schulz and associates (1995): i. Weightlifting experimental (WLeXp: $n=9$ ), or ii. Weightlifting placebo (WLPLA $\mathrm{n}=9$ ). All subjects were blinded to their group allocation. Subject characteristics are displayed in Table 1.

\subsection{Experimental approach}

The present study used a single-blinded, randomised, placebo-controlled study design. Every participant was given an appointment time and instructed to abstain from all forms of exercise for $48 \mathrm{~h}$ and fast for $2 \mathrm{~h}$ prior to attending their appointment. This controlled for prior physical stress and dietary intake affecting the study results (Carlson et al., 2008; 
Freidenreich \& Volek, 2012; Gleeson, 2007). Each participant attended the testing laboratory (temperature monitored at $23{ }^{\circ} \mathrm{C}$ ) on one single occasion only. The session began with a pre-screening where a Doctor of Physical Therapy confirmed that subjects were apparently healthy. Subjects were excluded based on: past or present history of upper limb/hand musculoskeletal pathology, cardiovascular, metabolic, or respiratory illness; contraindications to resistanceexercise (Pollock et al., 2000). Subjects were then familiarised with the $B \& L$ Pinch Gauge (PG-60) dynamometer (B \& L Engineering, CA, USA) and the MVC (maximum voluntary contraction) assessment using the familiarisation procedure described previously (Szlezak et al., 2015). Briefly, participants were seated in a standardised testing position as recommended by the American Society of Hand Therapists (Fess \& Moran, 1981). Only the dominant hand was utilised for the purpose of achieving maximal efforts. Participants were instructed to perform 5 sub-maximal pinch efforts separated by 30 seconds rest before immediately completing a maximal-effort practice session identical to the testing session (see 2.3.1 MVC Assessment). This familiarisation procedure also ensured that any pre-fatiguing effect associated with this procedure was consistent across all research participants. Participants were also shown a copy of the Pain Visual Analogue Scale (VAS) and were instructed on how to complete it. Following familiarization, an Intravenous (IV) catheter was inserted into the median cubital vein of the participant's dominant hand and was left insitu for serial blood sampling. This sampling method promoted precise timing of blood collection, and avoided repeat needle-trauma which could affect results. Blood for lymphocyte assessment was collected directly into an EDTA Vacutainer (Becton Dickinson, NJ, USA) and analysed within 60 minutes. Blood for lactate analysis was drawn with a syringe from the IV catheter and analysed immediately. At each collection point, $5 \mathrm{ml}$ of blood was drawn and discarded before collecting sample blood and catheter lines were maintained through saline flushing. After the catheter insertion, the participant rested for 10 minutes in the testing chair before baseline measures were collected to negate any potential affect the catheterisation could have on subsequent measures. Baseline measures were then recorded in the following order: i. Lateral pinch (see 2.3.1 MVC Assessment); ii. Pain perception (see 2.3.4 Pain Assessment); iii. Blood collection for lymphocyte assessment (see 2.3.5 Lymphocyte Assessment); iv. Blood collection for lactate analysis (see 2.3.6 Blood Lactate Analysis). Following baseline testing, participants undertook either an Exercise Intervention (WLEXP and UT EXP groups) or a Placebo Intervention (WLPLA group). Once the interventions were completed, all participants immediately underwent the following rest and passive testing conditions in the order described: i. Pain Perception: immediately, 10 minutes, 20 minutes and 60 minutes post the interventions; ii. Blood collection for lymphocyte assessment: 20 minutes and 60 minutes post the interventions; iii. Blood collection for lactate analysis: 3 minutes, 10 minutes, 20 minutes and 60 minutes post the interventions. Of note, blood Lactate was first sampled at 3 minutes post-intervention to record peak concentrations (Goodwin, Harris, Hernández, \& Gladden, 2007; Vucetic, Mozek, \& Rakovac, 2015). Throughout the duration of the experiment, all subjects remained seated and were instructed against moving the tested limb to avoid any active recovery which could affect the results. IV catheters were removed after the final collection point. All blood samples and raw data were subsequently analysed and statistical analysis applied.

\subsection{Procedures}

\subsubsection{MVC Assessment}

Assessment occurred using a $B \& L$ Engineering pinch gauge (PG-60) with reported reliability and validity (Mathiowetz et al., 1985; Mathiowetz, Vizenor, \& Melander, 2000; Mathiowetz, Weber, Volland, \& Kashman,1984). All subjects undertook this assessment to determine their maximum lateral-pinch-strength of the dominant hand only. To eliminate testing bias, one researcher gave instructions and reset the dynamometer and a separate researcher viewed and recorded each test result. Subject encouragement during testing was standardised by using the verbal instructions described previously (Mathiowetz et al., 1984). Subjects were positioned in the standardised testing position (seated in a chair with arm rests and full back support, the shoulder adducted to neutral and neutrally rotated, elbow flexed at $90^{\circ}$, forearm in the neutral position, and wrist between $0^{\circ}$ and $30^{\circ}$ dorsiflexion and between $0^{\circ}$ and $15^{\circ}$ ulnar deviation [Fess \& Moran, 1981; Mathiowetz et al., 1985]). This also controlled for contribution from accessory muscles. Subjects were tested using the protocol previously described (Mathiowetz et al., 1985). During testing, subjects were instructed to give a maximal effort. Immediately after the first effort was completed, the dynamometer was reset and a second (and final) effort was performed. Values were recorded and later averaged.

\subsubsection{Exercise Intervention}

A previously described low-dose exercise protocol (Szlezak et al., 2015) formed the exercise intervention. Briefly, this involved performing a lateral pinch-manoeuvre in a standardised testing position (Fess \& Moran, 1981; Mathiowetz et al., 1985) and utilised a B \& L Engineering pinch gauge (PG-60). A lateral-pinch manoeuvre was selected to minimise the potential effects of inter-individual biomechanical variation and accessory muscle exertion on study results (Punsola-Izard et al., 2012). A total of 4 minutes total-work (isometric thumb resistance-exercise) was performed, divided into four 60-s work-intervals each separated by one, 60-s rest interval. The total time for the exercise intervention was 7 minutes. The first two work intervals were performed at 50\% MVC and the remaining two work intervals at 35\% MVC (where MVC was determined during the MVC Assessment).

Participants continuously monitored the pinch gauge during the work-intervals to ensure they constantly applied the correct load $(\mathrm{kg})$. The standardised command "keep the needle at your weight" was used by a researcher if the applied load changed from that specified (Szlezak et al., 2015). 


\subsubsection{Placebo Intervention}

WLPLA participants undertook the placebo intervention (previously described by Szlezak et al., 2015) which involved 4 work-intervals lasting 60-s separated by 60-s rest-intervals. In contrast to the exercise intervention, WLPLA subjects held the pinch gauge without applying any downward pressure. This was considered a placebo since subjects could not know if they were performing a low-dose exercise intervention or not. Body positioning in the placebo intervention was identical to the exercise intervention. Participants rested during the rest-interval as described in the exercise intervention. A total-time of 7 minutes elapsed during the placebo intervention to maintain consistency with the exercise intervention.

\subsubsection{Pain Assessment}

A pain VAS was employed to validly and reliably assess each participant's perception of exercise-related muscle pain (Douris et al., 2006; Hawker, Mian, Kendzerska, \& French, 2011; Sellwood, Brukner, Williams, Nicol, \& Hinman, 2007). An unmarked horizontal $100 \mathrm{~mm}$ line with the terminal descriptors "no pain" (score of 0 ) and "worst pain imaginable" (score of 10) was completed by the participants. Scoring occurred by measuring the distance (mm) on the $10-\mathrm{cm}$ line between the "no pain" anchor and the respondent's mark thus providing a range of scores from 0-100 (Jensen, Karoly, \& Braver, 1986).

\subsubsection{Lymphocyte Assessment}

Lymphocyte subpopulation analysis was performed using the BD FACSVerse flow cytometer and the Becton Dickinson Simultest ${ }^{\mathrm{TM}}$ IMK Lymphocyte kit for enumeration of B (CD19+) lymphocytes, helper/inducer T (CD3+CD4+) lymphocytes, suppressor/cytotoxic T (CD3+CD8+) lymphocytes and natural killer (CD16+and/or CD56+) lymphocytes (Becton Dickinson, Franklin Lakes, NJ, USA). Lymphocyte cell percentages and absolute counts were calculated and generated automatically on each blood sample using the Simultest IMK-Lymphocyte software once the operator entered the independent data (leukocyte count, leukocyte and lymphocyte percentages; HmX Hematology analyser, Beckman Coulter, Pasadena, CA, USA).

\subsubsection{Blood Lactate Analysis}

Analysis occurred using an automated blood lactate analyser (Lactate Pro, Arkray Inc., Tokyo, JPN) with reported reliability and accuracy (Pyne, Boston, Martin, \& Logan, 2000). The device was calibrated prior to use as specified by the manufacturer. Venous blood was injected into a Lactate Pro test strip direct from the syringe. The analyser was left resting on a stationary table until the blood lactate calculation was finalised and recorded. Values were reported in millimoles per liter (mmol/L).

\subsection{Statistical Analysis}

Values presented are as means \pm standard deviation. IBM SPSS Statistics 22 was used for all statistical testing. Fullyfactorial ANOVA with repeated measures was used to determine any interaction among, or main effect of, the independent variables group and time. Least squares difference pairwise comparisons were used to detect the specific site of any significant effect identified. Pearson's correlation coefficients were calculated to determine any relationships among change in blood lactate concentration and change in lymphocyte subpopulation count for each of the participant groups (WLPLA, UT EXP, WLEXP). Statistical significance was accepted at $\mathrm{p}<0.05$.

\section{Results}

Subject characteristics are displayed in Table 1. The results of a one-way ANOVA revealed that no statistical differences were found for age $(\mathrm{F}=0.114, \mathrm{p}=0.892)$, height $(\mathrm{F}=0.561, \mathrm{p}=0.578)$ and body mass $(\mathrm{F}=1.096, \mathrm{p}=0.350)$ variables among the three groups. Moreover, no differences among the three groups were found for any of the measured variables (reported pain, blood lactate, lymphocyte counts) at baseline ( $p>0.05)$.

Table 1. Subject characteristics

\begin{tabular}{llll}
\hline \multicolumn{1}{c}{ Group } & \multicolumn{1}{c}{ UT $_{\text {EXP }}$} & \multicolumn{1}{c}{ WLEXP } & \multicolumn{1}{c}{ WLPLA } \\
\hline $\mathrm{n}$ & \multicolumn{1}{c}{9} & \multicolumn{1}{c}{9} & \multicolumn{1}{c}{9} \\
\hline Age $(\mathrm{yrs})$ & $27( \pm 7)$ & $28( \pm 5)$ & $27( \pm 7)$ \\
\hline Height $(\mathrm{cm})$ & $180( \pm 9)$ & $182( \pm 8)$ & $179( \pm 5)$ \\
\hline Body mass $(\mathrm{kg})$ & $83.2( \pm 9.8)$ & $89.6( \pm 7.4)$ & $84.3( \pm 11.6)$ \\
\hline Gym sessions (per week) & $0( \pm 0)$ & $4( \pm 1)$ & $4( \pm 1)$ \\
\hline Hand dominance & $\mathrm{R}: \mathrm{n}=8$ & $\mathrm{R}: \mathrm{n}=7$ & $\mathrm{R}: \mathrm{n}=7$ \\
& $\mathrm{~L}: \mathrm{n}=1$ & $\mathrm{~L}: \mathrm{n}=2$ & $\mathrm{~L}: \mathrm{n}=2$ \\
\hline Lateral pinch $(\mathrm{kg})$ & $8.7( \pm 1.5)$ & $9.7( \pm 1.2)$ & $8.7( \pm 1.5)$ \\
\hline \multicolumn{4}{l}{ Values presented are as means \pm standard deviation } \\
\hline
\end{tabular}

\subsection{Pain VAS}

A significant interaction for time and group was found for pain (VAS) $(\mathrm{F}=11.27, \mathrm{p}<0.01)$. Pairwise Comparisons revealed no significant change in the Placebo group from baseline at any post-intervention time-point $(\mathrm{p}>0.05)$. The UT $_{\text {EXP }}$ group increased significantly in reported pain immediately post-intervention $(2.92 / 10 ; p<0.01)$ and this represented the peak pain response. Pain remained elevated up to 20 minutes post-intervention $(p<0.01)$, before decreasing to levels not significantly different from baseline $(p>0.05)$ at 60 minutes post-intervention. The WLEXP group also reported a highly significant increase in pain immediately post-intervention $(4.92 / 10 ; \mathrm{p}<0.01)$ and this was the peak 
pain response. Pain remained elevated across all times points $(\mathrm{p}<0.01)$. Maximum pain values were significantly higher in the WLEXP $(4.92 / 10)$ vs $\operatorname{UT}_{\text {EXP }}(2.92 / 10)$ group $(\mathrm{p}=0.02)$.

3.2 Blood lactate

A significant interaction for time and group was found for blood lactate $(\mathrm{mmol} / \mathrm{L})(\mathrm{F}=26.31, \mathrm{p}<0.01)$. Pairwise Comparisons revealed no significant change in the Placebo group from baseline at any post-intervention time-point $(p>0.05)$. Both experimental groups showed a highly significant increase $(p<0.01)$ in blood lactate concentration at the first post-intervention ( 3 minutes post) and 10 minutes post-intervention time-points before returning to levels not significantly different from baseline by 20 minutes post-intervention $(p>0.05)$. Peak blood lactate concentrations (UT EXP: $2.2 \mathrm{mmol} / \mathrm{L}$; WLEXP: $2.4 \mathrm{mmol} / \mathrm{L}$ ) occurred at 3 minutes post-intervention for both experimental groups and response magnitude was not significantly different between these groups $(\mathrm{p}>0.05)$. See Figure 1.

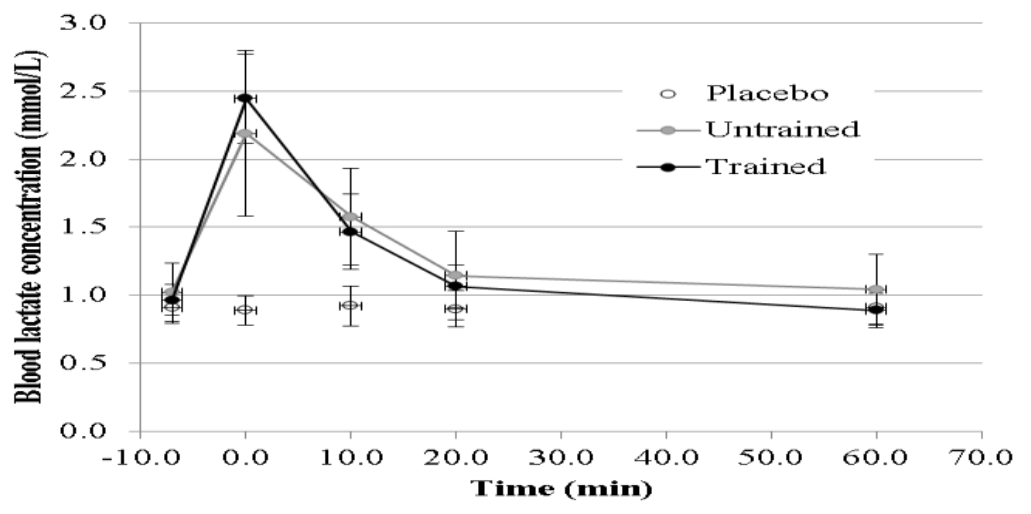

Figure 1. Blood lactate concentration over time

There was no difference in blood lactate concentration among the three groups at baseline ( -7 min; p>0.05). WLPLA: Lactate concentration did not change from baseline across time $(\mathrm{p}>0.05)$.

UT EXP and WLEXP: Lactate concentration increased $(\mathrm{p}<0.01)$ by $3 \mathrm{~min}$ post-exercise, remained elevated at $10 \mathrm{~min}$ postexercise $(\mathrm{p}<0.01)$, and decreased to levels not different from baseline by 20 min post-exercise $(p>0.05)$. Peak concentrations (3 min post: UT EXP: $2.2 \mathrm{mmol} / \mathrm{L}$; WLEXP: $2.4 \mathrm{mmol} / \mathrm{L}$ ) were not different between these two groups $(\mathrm{p}>0.05)$.

3.3 Helper/inducer T lymphocytes (CD4+CD3+)

A significant interaction for time and group was found for $\mathrm{CD} 4+\mathrm{CD} 3+$ lymphocytes $(\mathrm{F}=4.42, \mathrm{p}<0.01)$. Pairwise Comparisons revealed no significant change in the Placebo group from baseline at any post-intervention time-point ( $\mathrm{p}>0.05$ ). In the $\mathrm{UT}_{\mathrm{EXP}}$ group, lymphocyte count increased from baseline at 20 minutes post-intervention by $20.78 \%$ and this change was highly significant $(\mathrm{p}<0.01)$. From 20-60 minutes post-intervention, lymphocyte count decreased back towards baseline count and this was also highly significant $(\mathrm{p}<0.01)$. Lymphocyte count at the final time-point $(60$ minutes post-intervention) was not significantly different from baseline ( $p>0.05)$.

In the WLeXP group, lymphocyte count did not significantly change between baseline and 20 minutes post-intervention ( $p>0.05$ ). Lymphocyte count did elevate from 20-60 minutes post-intervention and this change was significant $(\mathrm{p}=0.01)$. Lymphocyte count at the final time-point was elevated above baseline by $19.06 \%$ and this difference was highly significant $(\mathrm{p}<0.01)$. See Figure 2.

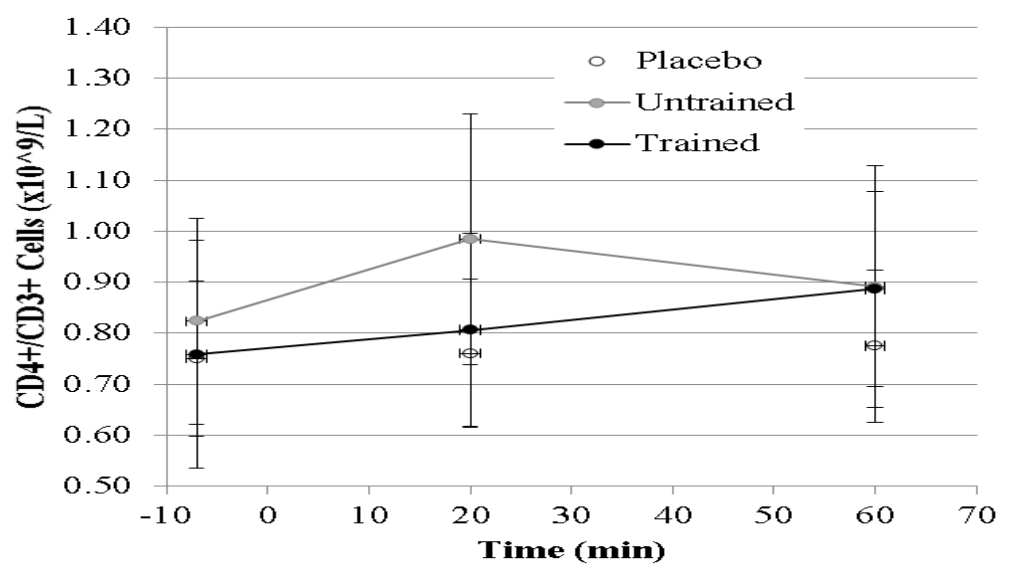

Figure 2. CD4+CD3+ T lymphocyte count over time

There was no difference in CD4+CD3+ cell count among the three groups at baseline ( $-7 \mathrm{~min} ; \mathrm{p}>0.05)$.

WLPLA: Lymphocyte count did not change from baseline across time ( $\mathrm{p}>0.05)$.

UTEXP: Lymphocyte count had increased by 20 min post-exercise $(\Delta 20.78 \%$; $p<0.01)$, then decreased from $20-60 \mathrm{~min}$ post $(\mathrm{p}<0.01)$. At $60 \mathrm{~min}$ post-exercise, lymphocyte count was not different from baseline $(\mathrm{p}>0.05)$.

WLexp: Lymphocyte count had not changed by 20 min post-exercise (p>0.05) but increased from 20-60 min postexercise $(\mathrm{p}=0.01)$. At $60 \mathrm{~min}$ post-exercise, lymphocyte count was elevated above baseline $(\Delta 19.06 \% ; \mathrm{p}<0.01)$.

3.4 Suppressor/cytotoxic T lymphocytes $(C D 8+C D 3+)$ 
A significant interaction for time and group was found for $C D 8+C D 3+$ lymphocytes $(F=3.1, p=0.02)$. Pairwise Comparisons revealed no significant change in the Placebo group from baseline at any post-intervention time-point (p>0.05).In the $\mathrm{UT}_{\text {EXP }}$ group, lymphocyte count increased from baseline at 20 minutes post-intervention by $15.25 \%$ and this change was highly significant $(\mathrm{p}<0.01)$. From 20-60 minutes post-intervention, lymphocyte count decreased back towards baseline count and this was also significant $(\mathrm{p}=0.03)$. Lymphocyte count at the final time-point (60 minutes post-intervention) was not significantly different from baseline ( $p>0.05)$.

In the $\mathrm{WL}_{\mathrm{EXP}}$ group, lymphocyte count did not significantly change between baseline and 20 minutes post-intervention ( $p>0.05$ ). Lymphocyte count did increase from 20-60 minutes post-intervention however this change did not reach significance ( $>0.05$ ). Lymphocyte count at the final time-point had elevated above baseline by $11.46 \%$ and this difference was significant ( $\mathrm{p}=0.033)$. See Figure 3.

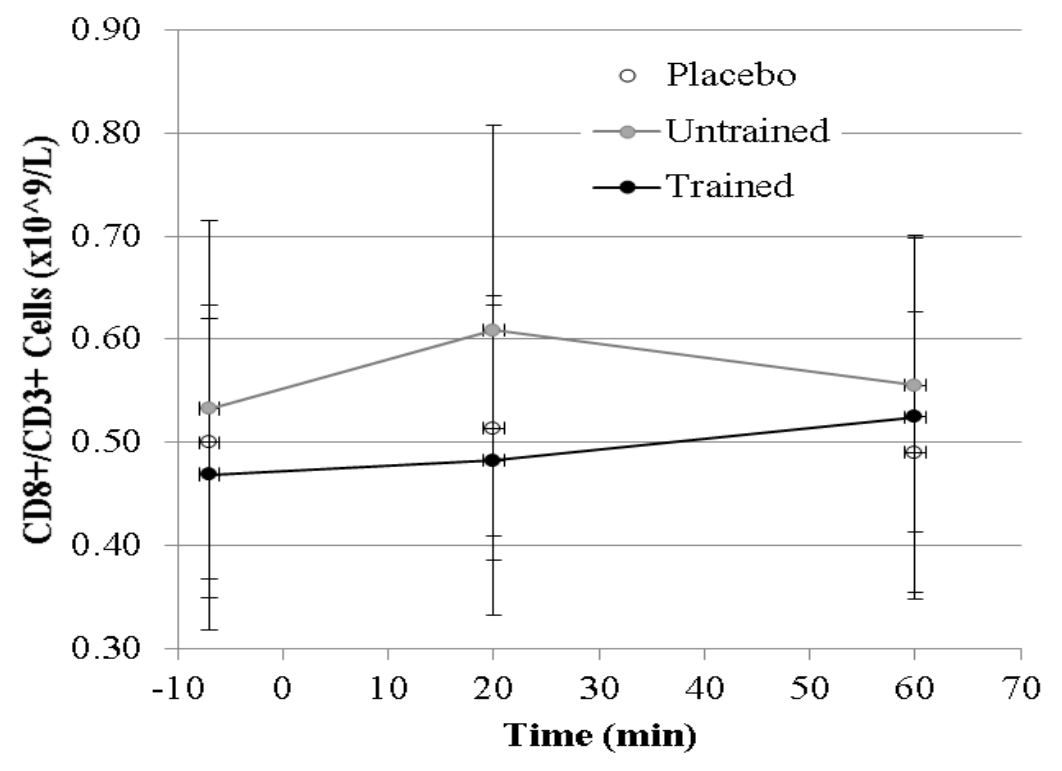

Figure 3. CD8+CD3+ T lymphocyte count over time

There was no difference in CD8+CD3+ cell count among the three groups at baseline ( $-7 \mathrm{~min} ; \mathrm{p}>0.05)$.

WLPLA: Lymphocyte count did not change from baseline across time $(\mathrm{p}>0.05)$.

UT ExP: Lymphocyte count had increased by $20 \mathrm{~min}$ post-exercise $(\Delta 15.25 \%$; $<<0.01)$, then decreased from $20-60 \mathrm{~min}$ post-exercise ( $p=0.03)$. At $60 \mathrm{~min}$ post-exercise, lymphocyte count was not different from baseline ( $p>0.05)$.

WLEXP: Lymphocyte count had not changed by 20 min post-exercise ( $p>0.05)$. Lymphocyte count did increase from 20-60 min post-exercise however this change did not reach significance ( $p>0.05)$. At 60 min post-exercise, lymphocyte count was elevated above baseline $(\Delta 11.46 \%, \mathrm{p}=0.033)$.

\subsection{B lymphocytes (CD19+)}

No interaction of time and group was found for $\mathrm{CD} 19+$ lymphocytes $(\mathrm{F}=1.72, \mathrm{p}=0.16)$ however there was a main effect of time $(\mathrm{F}=6.86, \mathrm{p}<0.01)$. Pairwise comparisons revealed no significant change in the Placebo group from baseline at any post-intervention time-point ( $\mathrm{p}>0.05)$. In the $\mathrm{UT}_{\mathrm{EXP}}$ group, lymphocyte count increased from baseline at 20 minutes post-intervention by $18.11 \%$ and this change was significant $(\mathrm{p}=0.013)$. From $20-60$ minutes post-intervention, lymphocyte count decreased back towards baseline count however this change did not reach significance ( $p>0.05)$. Lymphocyte count at the final time-point (60 minutes post-intervention) was not significantly different from baseline $(p>0.05)$. In the $W L_{E X P}$ group, lymphocyte count did not significantly change between baseline and 20 minutes postintervention $(p>0.05)$. Lymphocyte count increased from 20-60 minutes post-intervention and this change was significant $(\mathrm{p}=0.031)$. Lymphocyte count at the final time-point was elevated above baseline by $28.87 \%$ and this difference was highly significant $(\mathrm{p}<0.01)$. See Figure 4. 


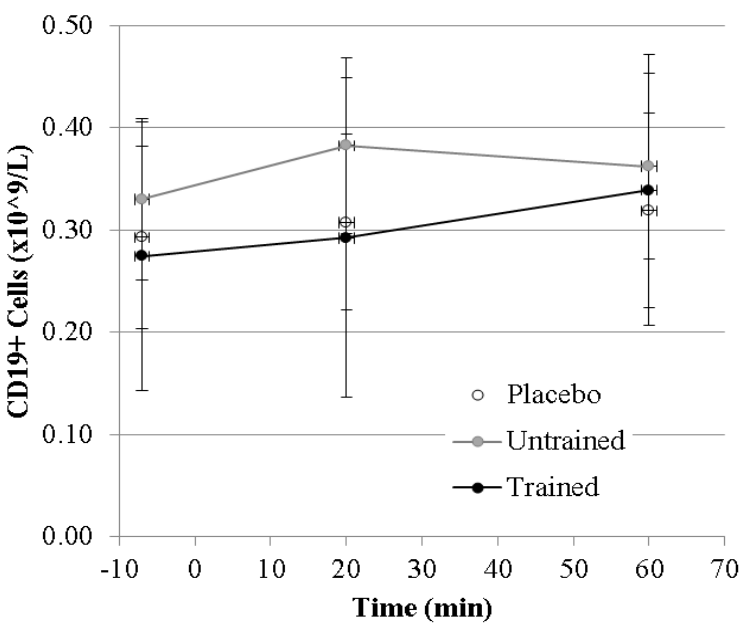

Figure 4. CD19+ B lymphocyte count over time

There was no difference in CD19+ cell count among the three groups at baseline (-7 min; $\mathrm{p}>0.05)$.

WLPLA: Lymphocyte count did not change from baseline across time ( $p>0.05)$.

UTEXP: Lymphocyte count had increased by 20 min post-exercise $(\Delta 18.11 \%$; $=0.013)$. A decrease occurred from 20 $60 \mathrm{~min}$ post-exercise but did not reach significance $(\mathrm{p}>0.05)$. At $60 \mathrm{~min}$ post-exercise, lymphocyte count was not different from baseline ( $\mathrm{p}>0.05)$.

WLexp: Lymphocyte count had not changed by 20 min post-exercise (p>0.05) but increased from 20-60 min postexercise $(\mathrm{p}=0.031)$. At $60 \mathrm{~min}$ post-exercise, lymphocyte count was elevated above baseline $(\Delta 28.87 \% ; \mathrm{p}<0.01)$.

\subsection{Natural Killer lymphocytes (CD56+ and or CD16+)}

No significant interactions were found for time and groups for CD56+/CD16+ lymphocytes $(\mathrm{F}=0.23, \mathrm{p}=0.92)$. Additionally, no significant changes in lymphocyte count from baseline occurred across time for any of the three groups $(\mathrm{p}>0.05)$. See Figure 5.

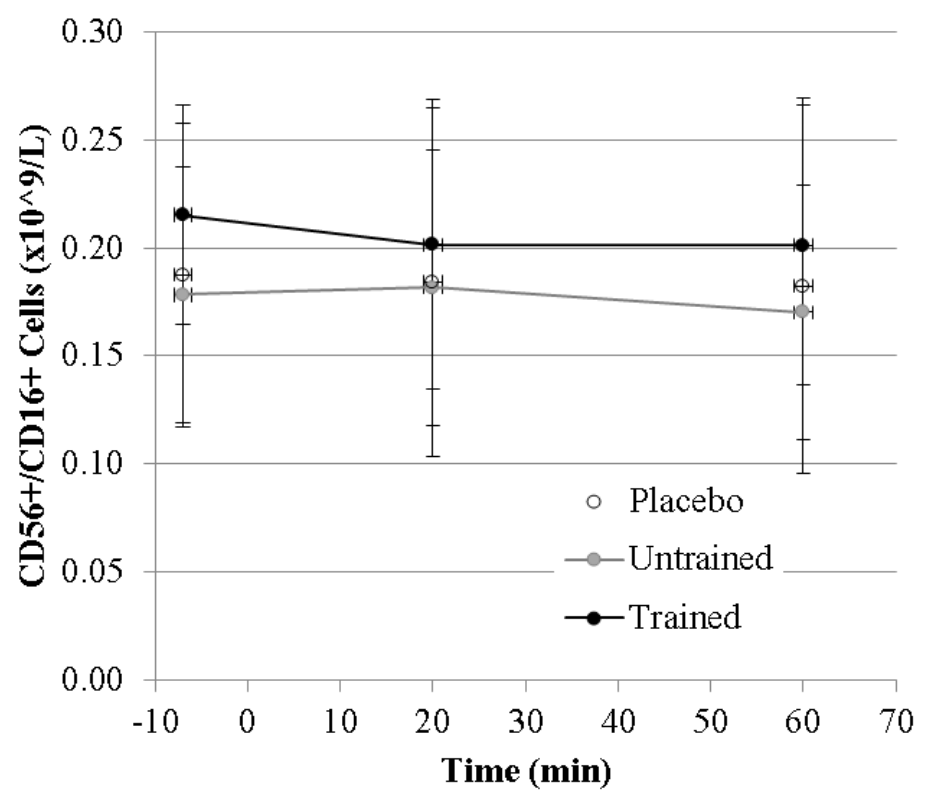

Figure 5. CD56+/CD16+ NK lymphocyte count over time

There was no difference in CD56+/CD16 cell count among the three groups at baseline $(-7 \mathrm{~min} ; \mathrm{p}>0.05)$.

No changes in lymphocyte count from baseline occurred across time for any of the three groups $(\mathrm{p}>0.05)$.

\subsection{Blood lactate - lymphocyte correlation}

No correlation was found between change in blood lactate concentration and change in lymphocyte count: CD4+CD3+ $\left(\mathrm{UT}_{\text {EXP }} \mathrm{r}=0.11, \mathrm{p}=0.78\right.$; WLEXP $\left.\mathrm{r}=0.16, \mathrm{p}=0.68\right), \mathrm{CD} 8+\mathrm{CD} 3+\left(\mathrm{UT}_{\text {EXP }} \mathrm{r}=0.03, \mathrm{p}=0.93\right.$; WL $\left.\mathrm{EXP}_{\mathrm{r}} \mathrm{r}=0.64, \mathrm{p}=0.07\right), \mathrm{CD} 19+$ $\left(\mathrm{UT}_{\text {EXP }} \mathrm{r}=-0.05, \mathrm{p}=0.89\right.$; WLEXP $\mathrm{r}=0.21, \mathrm{p}=0.60$ ) or CD56+/16+ cell counts $\left(\mathrm{UT}_{\text {EXP }} \mathrm{r}=0.20, \mathrm{p}=0.76\right.$; WLEXP $\mathrm{r}=-0.01$, $\mathrm{p}=0.99$ ). The relationship observed between blood lactate concentration and CD8+CD3+ cell count in WLEXP is illustrated in Figure 6; nonetheless this relationship was not statistically significant. 


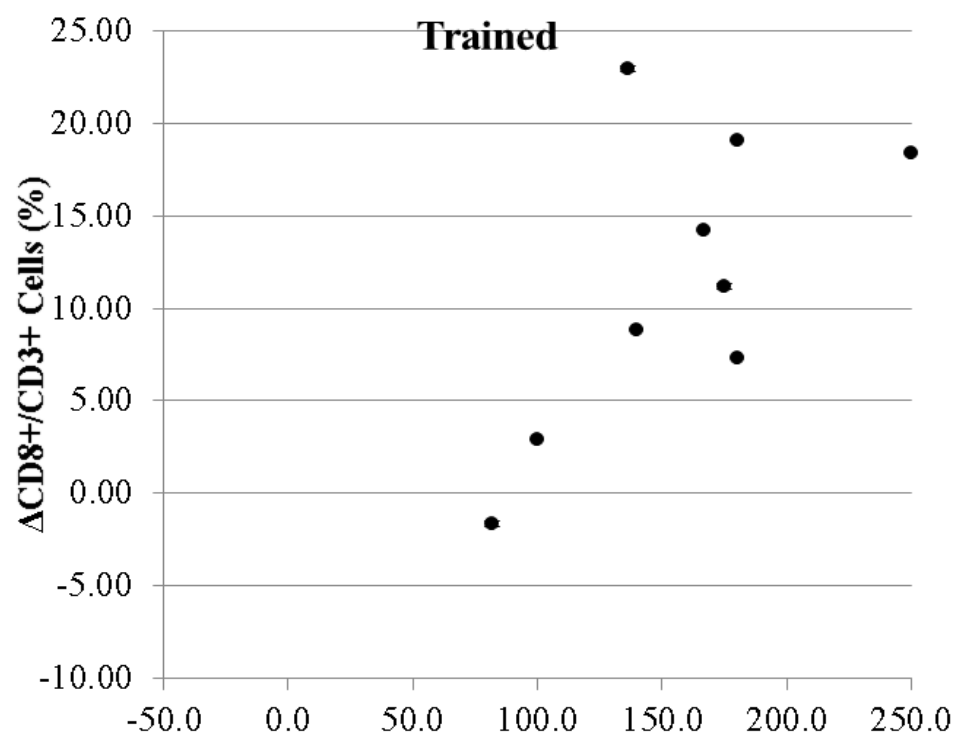

$\Delta$ Blood lactate concentration (\%)

Figure 6. Blood lactate concentration \& CD8+CD3+ lymphocyte correlation (WLEXP)

The relationship observed between changes in blood lactate concentration and changes in CD8+CD3+ cell count in $\mathrm{WL}_{\text {EXP }}(\mathrm{r}=0.64)$ is illustrated. Nonetheless, this relationship was not significant $(\mathrm{p}>0.05)$.

\section{Discussion}

The present study is the first to demonstrate that a micro-dose of resistance-exercise can significantly elevate circulating B lymphocyte and T lymphocyte counts. Additionally, lymphocyte response profiles were related to the physical aptitude of subjects, and not blood lactate levels. No relationship was found between change in lymphocyte counts (CD4+CD3+ and CD8+CD3+ T cells; CD19+ B cells; CD56+/16+ NK cells) and change in blood lactate concentration for either of the experimental groups. This latter finding disproved our hypothesis that an increase in circulating lymphocyte count is related to an increase in blood lactate concentration. Functionally, these findings indicate that B and $\mathrm{T}$ cell trafficking in males is affected by even the smallest of physical exertions. Moreover, cell mobilisation is not regulated by mild elevations in cellular energy demand or anaerobic energy expenditure (Fernandez et al., 2006; Wirtz et al., 2014) however is influenced by physical aptitude of the host.

The present study utilised a micro-dose exercise approach which was brief in duration, low in intensity and anaerobically based (evidenced by increases in blood lactate as hypothesised). This experimental approach was considered critical in order to minimise the potential influence of exercise intensity and duration on the study results (Freidenreich \& Volek, 2012; Walsh et al., 2011) so that potential relationships between blood lactate, physical aptitude and lymphocyte homeostasis could be better studied. It was also necessary to investigate the effects of a low-dose resisted exertion on lymphocyte subpopulations.

In the present study, the placebo group did not change for any measured variable across any time-points as expected. For reported pain levels, the significant increase seen for the UTEXP and WLEXP groups indicates that a low intensity, short duration exercise protocol can be highly challenging beyond the physical, even in a well-trained athletic population. Moreover, the pain elevation was shortly lived ( $<20$ minutes) in the untrained individuals, whereas the response was maintained for at least 60 minutes (final time-point) in the weightlifters. Of note, the weightlifters reported a significantly higher peak pain response compared with the untrained group. As peak blood lactate concentrations between groups were not significantly different, a psychological phenomenon may be responsible for this result. Well trained athletes are known to place higher expectations on oneself than non-athletes (Dunn, Gotwals, \& Causgrove Dunn, 2005; Gould, Dieffenbach, \& Moffett, 2002) which can be associated with perfectionistic concerns, fear of failure, mental stress and elevated anxiety (Conroy, Willow, \& Metzler, 2002; Stoeber et al., 2011). These alterations in psychological state are known to increase one's perception of pain (Loggia, Schweinhardt, Villemure, \& Bushnell, 2008; Rhudy \& Meagher, 2000) and likely explain the results of this present study. The magnitude of peak pain reported in this study for the weightlifting subjects (4.92/10) was a novel and unexpected finding considering the micro-dose nature of the exercise protocol. This is evidence that low-intensity, short-duration exercise can be highly demanding depending on the subject population and the targeted muscle groups.

Blood lactate concentrations increased significantly in both experimental groups at 3 minutes post-exercise and had normalised by 20 minutes post-exercise, indicating a similar production and clearance rate between experimental groups. The timing of the peak concentration (3 minutes post-exercise) was consistent with previously reported temporal profiles in larger-dose studies (Goodwin et al., 2007; Vucetic et al., 2015). Interestingly, lactate profiles between experimental groups were similar and may be explained by the involvement of the thumb muscle in activities of daily living, thus providing a training effect in the untrained group. Importantly, these findings indicated that the 
exercise-intervention challenged each experimental group to a similar level, both physically and anaerobically. The blood lactate profile demonstrated in the present study is a novel and meaningful finding alone. These results show that short duration exercise at a low physical intensity (35\% and 50\% of MVC) can measurably increase anaerobic energy expenditure in specific skeletal muscle (Fernandez et al., 2006; Wirtz et al., 2014). For example, a large muscle exercising at 35\% MVC may be below lactate threshold for that tissue, however for the small muscle group around the thumb, this same relative intensity was above the lactate threshold. These results show how a low physical intensity of exercise can have meaningful metabolic consequences for specific myocytes. Extrapolating the results of the present study to a practical example, gripping an unloaded barbell would not be considered challenging for a trained athlete, however the coach should now consider the metabolic implications for the smaller gripping muscles, those in the thumb and hand. Additionally, we have shown that a small muscle group exertion can produce high levels of pain, thus researchers and clinicians should view exercise intensity in a broader scope than just the physical.

In relation to lymphocyte subpopulations, no significant change was seen for the CD56+/CD16+ cells. This negative result was inconsistent with the literature (Freidenreich \& Volek, 2012), however as the first measurement was not taken until 20 minutes post-exercise, a response may have occurred and returned to baseline prior to this point. As this study is the first to investigate natural killer cell responses to such a short duration, micro-dose of resistance-exercise, further research should measure natural killer cell counts at more acute post-exercise time-points. Conversely, CD4+CD3+ cells, CD8+CD3+ cells and CD19+ cells all demonstrated a characteristic response pattern to exercise associated with training status. In the UTEXP group, the cell count (circulating) for these cell types had significantly increased at the first post-intervention time-point (20 minutes post-intervention) then significantly decreased back to values not different from baseline by the final time-point. In contrast, a completely different response profile was observed for the WLEXP group. In the WLEXP group, the same cell types showed a delayed increase. No significant increase was seen at the first post-intervention time-point, however cell counts were significantly elevated above baseline at the final time-point.

To reiterate, untrained individuals showed an early increase then a decrease in lymphocyte count, whereas weightlifting trained individuals showed no early increase followed by a delayed increase. Of note, the magnitude in lymphocyte responses were smaller than previously reported data related to high-dose exercise protocols (Freidenreich \& Volek, 2012; Walsh et al., 2011), suggesting that a dose-response relationship does exist for exercise-associated lymphocytosis. However, the lymphocyte responses observed in the present study provide strong evidence that exercise intensity and duration alone do not dictate the response to exercise, as both experimental groups undertook a common exercise protocol yet responded uniquely. Another major finding of the present study is the lack of correlation between blood lactate and lymphocyte subpopulation count as previously reported in females (Miles et al., 2003). This disagreement between previous observations and those of the present study are likely explainable though differences in study design. The earlier study (Miles et al., 2003) found that high lactate responders ( $\geq 11.92 \mathrm{mmol} / \mathrm{L})$ showed significantly greater lymphocyte count elevations than lower lactate responders $(\leq 7.62 \mathrm{mmol} / \mathrm{L})$. In contrast, the present study's low dose exercise protocol induced lower levels of lactate production $(\leq 2.4 \mathrm{mmol} / \mathrm{L})$ despite significant lymphocyte count elevation. Additionally, the present study employed all-male subjects whereas Miles and associates (2003) used only females. Also, the results previously reported (Miles et al., 2003) were based on performance of a high-dose multiplejoint (squat) exercise protocol. In consideration of potential inter-individual biomechanical differences (Frank et al., 2013; Solomonow \& Krogsgaard, 2001), standardisation of exercise performance and thus reliability of study results cannot be guaranteed in this latter study. Conversely, the present study utilised a highly controllable exercise intervention with strict standardisation of body positioning, skeletal joint and muscle involvement (Mathiowetz et al., 1985; Punsola-Izard et al., 2012; Towles, Hentz, \& Murray, 2008). Finally, the present study included both well-trained and untrained subjects whereas the previous study recruited previously-untrained subjects only. If lymphocyte redistribution was related to mild elevations in blood lactate concentration, strong correlations should have been calculated for both experimental groups for each of the four lymphocyte subpopulations (which was not the case). Importantly, blood lactate did not differ significantly between weightlifting trained and untrained individuals in the present study. That is, peak concentrations and temporal profiles were not significantly different between experimental groups, however their lymphocyte responses to resistance-exercise where markedly different. A unique lymphocyte response relative to training status was hypothesised, however this was based upon anticipated differences in anaerobic response between groups, which was not seen. This is novel evidence that mild elevations of blood lactate cannot alone explain the selective redistribution of lymphocytes into the circulation (in males). As the present study used a common exercise protocol which exerted all subjects to a similar level of anaerobic glycolytic activity, and considering that blood lactate was cleared at a similar rate, the only significant difference between the groups was the training status variable. From another perspective, the relationship between high blood lactate levels and lymphocyte counts in females (Miles et al., 2003) contrasted with the present study's findings give rise to the notion that lymphocytes may react to lactate above a certain threshold only. Future studies should investigate this suggestion through a stringently controlled exercise protocol which employs multiple anaerobic-doses.

Since blood lactate is associated with exercise-induced catecholamine changes (Pullinen, Nicol, MacDonald, \& Komi, 1999) and lymphocytes unlike other leukocytes are uniquely sensitivity to catecholamines (Benschop, Schedlowski, Wienecke, Jacobs, \& Schmidt, 1997; Landmann, 1992), it may be that catecholamines and not blood lactate are responsible for the characteristic lymphocyte profiles observed in the present study. This is plausible since resistancetraining is known to increase the magnitude of catecholamine release during exercise (Kraemer et al., 1999). Future 
researchers are encouraged to investigate if catecholamines are mechanistically responsible for the relationship between lymphocyte counts and training status post low-dose resistance-exercise. Additionally, lymphocyte functionality should now be examined post low-dose exercise to determine if the associated lymphocytosis carries clinically relevant implications.

\section{Conclusion}

The results of our study provide original evidence that significant changes to B and $\mathrm{T}$ lymphocyte homeostasis can occur in the absence of high intensity, high volume exercise. Moreover, resistance-exercise induced lymphocytosis cannot be explained by blood lactate when elevations are only mild. This finding does not rule out a potential relationship between blood lactate and lymphocyte trafficking, however suggests another mechanism may be involved at low exercise doses. Until the functional implications of these exercise-induced lymphocyte responses are determined, exercise-professionals are advised to recognise that any dose of resistance-exercise may affect lymphocyte trafficking. Whilst the influence of exercise intensity and duration on leukocyte responses to resistance-exercise have been extensively reported in the literature, our findings demonstrate that B and $\mathrm{T}$ lymphocytes specifically, respond to a lowdose of resistance-exercise in a characteristic manner relative to the physical aptitude of the host.

\section{References}

Azeem, K., \& Kumar, R. (2011). Effects of weight training on power performance. Journal of Physical Education and Sport, 11(2), 124-126.

Benschop, R. J., Schedlowski, M., Wienecke, H., Jacobs, R., \& Schmidt, R. E. (1997). Adrenergic control of natural killer cell circulation and adhesion. Brain, Behavior and Immunity, 11, 321-332. http://dx.doi.org/10.1006/brbi.1997.0499

Bush, J. A., Kraemer, W. J., Mastro, A. M., Triplett-McBride, N. T., Volek, J. S., Putukian, M., Sebastianelli, W. J., \& Knuttgen, H. G. (1999). Exercise and recovery responses of adrenal medullary neurohormones to heavy resistanceexercise. Medicine and Science in Sports and Exercise, 31, 554-559. http://dx.doi.org/10.1097/00005768-19990400000010

Carlson, L. A., Headley, S., DeBruin, J., Tuckow, A. T., Koch, A. J., \& Kenefick, R. W. (2008). Carbohydrate supplementation and immune responses after acute exhaustive resistance-exercise. International Journal of Sport Nutrition and Exercise Metabolism, 18(3), 247-259.

Conroy, D. E., Willow, J. P., \& Metzler, J. N. (2002). Multidimensional fear of failure measurement: The Performance Failure Appraisal Inventory. Journal of Applied Sport Psychology, 14(2), 76-90. http://dx.doi.org/10.1080/10413200252907752

Dohi, K., Mastro, A. M., Miles, M. P., Bush, J. A., Grove, D. S., Leach, S. K., Volek, J. S., Nindl, B. C., Marx, J. O., Gotshalk, L.A., Putukian, M., Sebastianelli, W. J., Kraemer, W. J. (2001). Lymphocyte proliferation in response to acute heavy resistance exercise in women: influence of muscle strength and total work. European Journal of Applied Physiology, 85(3-4), 367-373. http://dx.doi.org/10.1007/s004210100388

Douris, P., Southard, V., Ferrigi, R., Grauer, J., Katz, D., Nascimento, C., Podbielski, P. (2006). Effect of phototherapy on delayed onset muscle soreness. Photomedicine and Laser Surgery, 24(3), $377-382$. http://dx.doi.org/10.1089/pho.2006.24.377

Dunn, J. G. H., Gotwals, J. K., \& Causgrove Dunn, J. (2005). An examination of the domain specificity of perfectionism among intercollegiate student-athletes. Personality and Individual Differences, 38(6), 1439-1448. http://dx.doi.org/10.1016/j.paid.2004.09.009

Fernandez, J., Mendez-Villanueva, A., Pluim, B. M. (2006). Intensity of tennis match play. British Journal of Sports Medicine, 40(5), 387-391. http://dx.doi.org/10.1136/bjsm.2005.023168

Fess, E. E., \& Moran, C. (1981). Clinical assessment recommendations. American Society of Hand Therapists. Indianapolis.

Frank, C., Kobesova, A., \& Kolar, P. (2013). Dynamic neuromuscular stabilization \& sports rehabilitation. International Journal of Sports Physical Therapy, 8(1), 62-73.

Freidenreich, D. J., \& Volek, J. S. (2012). Immune responses to resistance-exercise. Exercise Immunology Review, 18, 8-41.

Gleeson, M. J. (2007). Immune function in sport and exercise. Journal of Applied Physiology, 103(2), $693-699$. http://dx.doi.org/10.1152/japplphysiol.00008.2007

Goodwin, M. L., Harris, J. E., Hernández, A., \& Gladden, B. L. (2007). Blood lactate measurements and analysis during exercise: A guide for clinicians. $J$ of Diabetes, Science and Technology, 1(4), 558-569. http://dx.doi.org/10.1177/193229680700100414

Gould, D., Dieffenbach, K., \& Moffett, A, (2002). Psychological characteristics and their development in Olympic champions. Journal of Applied Sport Psychology, 14(3), 172-204. http://dx.doi.org/10.1080/10413200290103482

Hawker, G. A., Mian, S., Kendzerska, T., \& French, M. (2011). Measures of Adult Pain, Visual Analog Scale for Pain (VAS Pain), Numeric Rating Scale for Pain (NRS Pain), McGill Pain Questionnaire (MPQ), Short-Form McGill Pain 
Questionnaire (SF-MPQ), Chronic Pain Grade Scale (CPGS), Short Form-36 Bodily Pain Scale (SF-36 BPS), and Measure of Intermittent and Constant Osteoarthritis Pain (ICOAP). Arthritis Care and Research, 63(11), S240-S252. http://dx.doi.org/10.1002/acr.20543

Jensen, M. P., Karoly, P., \& Braver, S. (1986). The measurement of clinical pain intensity: a comparison of six methods. Pain, 27(1), 117-126. http://dx.doi.org/10.1016/0304-3959(86)90228-9

Kraemer, W. J., Fleck, S. J., Maresh, C. M., Ratamess, N. A., Gordon, S. E., Goetz, K. L., Harman, E. A., Frykman, P. N., Volek, J. S., Mazzetti, S. A., Fry, A. C., Marchitelli, L. J., \& Patton, J. F. (1999). Acute hormonal responses to a single bout of heavy resistance-exercise in trained power lifters and untrained men. Canadian Journal of Applied Physiology, 24, 524-537.

Landmann, R. (1992). Beta-adrenergic receptors in human leukocyte subpopulations. European Journal of Clinical Investigation, 22(Suppl 1), 30-36.

LeBien, T. W., \& Tedder, T. F. (2008). B lymphocytes: how they develop and function. Blood, 112(5), 1570-1580. http://dx.doi.org/10.1182/blood-2008-02-078071

Luckheeram, R. V., Zhou, R., Verma, A. D., \& Xia, B. (2012). CD4 ${ }^{+}$T cells: differentiation and functions. Clinical and Developmental Immunology, 2012, 925135. http://dx.doi.org/10.1155/2012/925135

Loggia, M. L., Schweinhardt, P., Villemure, C., Bushnell, M. C. (2008). Effects of psychological state on pain perception in the dental environment. Journal (Canadian Dental Association), 74(7), 651-656.

Mathiowetz, V., Kashman, N., Volland, G., Weber, K., Dowe, M., \& Rogers, S. (1985). Grip and pinch-strength: normative data for adults. Archives of Physical Medicine and Rehabilitation, 66(2), 69-74.

Mathiowetz, V., Vizenor, L., \& Melander, D. (2000). Comparison of Baseline Instruments to the Jamar Dynamometer and the B\&L Engineering Pinch Gauge. Occupational Therapy Journal of Research, 20(3),147-162. http://dx.doi.org/10.1177/153944920002000301

Mathiowetz. V., Weber, K., Volland, G., \& Kashman, N. (1984). Reliability and validity of grip and pinch-strength evaluations. The Journal of Hand Surgery, 9(2), 222-226.

Mayhew, D. L., Thyfault, J. P., \& Koch, A. J. (2005). Rest-interval length affects leukocyte levels during heavy resistance-exercise. Journal of Strength and Conditioning Research, 19,16-22. http://dx.doi.org/10.1519/00124278200502000-00004

Miles, M. P., Kraemer, W. J., Nindl, B. C., Grove, D. S., Leach, S. K., Dohi, K., Marx, J. O., Volek, J. S., \& Mastro, A. M. (2003). Strength, workload, anaerobic intensity and the immune response to resistance exercise in women. Acta Physiologica Scandinavica, 178(2), 155-163. http://dx.doi.org/10.1046/j.1365-201x.2003.01124.x

Mosser, D. M., \& Edwards, J. P. (2008). Exploring the full spectrum of macrophage activation. Nature Reviews Immunology, 8, 958-969. http://dx.doi.org/10.1038/nri2788

Pollock, M. L., Franklin, B. A., Balady, G. J., Chaitman, B. L., Fleg, J. L., Fletcher, B., Limacher, M., Piña, I. L., Stein, R. A., Williams, M., \& Bazzarre, T. (2000). Resistance-exercise in individuals with and without cardiovascular disease: benefits, rationale, safety, and prescription. An advisory from the committee on exercise, rehabilitation, and prevention, council on clinical cardiology, American Heart Association. Circulation, 101, 828-833. http://dx.doi.org/10.1161/01.cir.101.7.828

Pullinen, T., Nicol, C., MacDonald, E., \& Komi, P. V. (1999). Plasma catecholamine responses to four resistance exercise tests in men and women. European Journal of Applied Physiology and Occupational Physiology, 80, 125-131. http://dx.doi.org/10.1007/s004210050568

Punsola-Izard, V., Salas-Gómez, D., Sirvent-Rivalda, E., \& Esquirol-Caussà, J. (2012). Functional patterns of thumb key pinch and their influence on thumb strength and stability. Hand Therapy, 17(4), 78-86. http://dx.doi.org/10.1258/ht.2012.012016

Pyne, D. B., Boston, T., Martin, D.T., \& Logan, A. (2000). Evaluation of the Lactate Pro blood lactate analyser. European Journal of Applied Physiology, 82(1-2), 112-116. http://dx.doi.org/10.1007/s004210050659

Ranisavljev, I., \& Vladimir, I. (2010). Modalities of training parameter alternation in nowadays strength training practice. Journal of Physical Education and Sport, 29(4), 41-46.

Rhudy, J. L., \& Meagher, M. W. (2000). Fear and anxiety: divergent effects on human pain thresholds. Pain, 84(1), 6575. http://dx.doi.org/10.1016/s0304-3959(99)00183-9

Schulz, K. F., Chalmers, I., Hayes, R. J., \& Altman, D. G. (1995). Empirical evidence of bias: Dimensions of methodological quality associated with estimates of treatment effects in controlled trials. Journal of the American Medical Association, 273(5), 408-412. http://dx.doi.org/10.1001/jama.273.5.408

Sellwood, K. L., Brukner, P., Williams, D., Nicol, A., \& Hinman, R. (2007). Ice-water immersion and delayed-onset muscle soreness: a randomised controlled trial. British Journal of Sports Medicine, 41(6), 392-397. http://dx.doi.org/10.1136/bjsm.2006.033985

Solomonow, M., \& Krogsgaard, M. (2001). Sensorimotor control of knee stability. A review. Scandinavian Journal of Medicine and Science in Sports, 11(2), 64-80. http://dx.doi.org/10.1034/j.1600-0838.2001.011002064.x 
Sonnet, C., Lafuste, P., Arnold, L., Brigitte, M., Poron, F., Authier, F. J., Chretien, F., Gherardi, R. K., \& Chazaud, B. (2006). Human macrophages rescue myoblasts and myotubes from apoptosis through a set of adhesion molecular systems. Journal of Cell Science, 119, 2497-2507. http://dx.doi.org/10.1242/jcs.02988

Spurway, N. C. (1992) Aerobic exercise, anaerobic exercise and the lactate threshold. British Medical Bulletin, 48(3), $569-91$.

Stoeber, J. (2011). The dual nature of perfectionism in sports: Relationships with emotion, motivation, and performance. International Review of Sport and Exercise Psychology, 4(2), 128-145s. http://dx.doi.org/10.1080/1750984x.2011.604789

Szlezak, A. M., Tajouri, L., Keane, J., Szlezak, S. L., Minahan, C. (2015). Micro-dose of resistance-exercise: effects of sub-maximal thumb exertion on leukocyte redistribution and fatigue in trained male weightlifters. Journal of Physical Education and Sport, 15(3), 365-377. http://dx.doi.org/10.7752/jpes.2015.03055

Towles, J. D., Hentz, V. R., \& Murray, W. M. (2008). Use of intrinsic thumb muscles may help to improve lateral pinch function restored by tendon transfer. Clinical Biomechanics, 23(4), 387-394. http://dx.doi.org/10.1016/j.clinbiomech.2007.11.008

Vucetic, V., Mozek, M., \& Rakovac, M. (2015). Peak blood lactate parameters in athletes of different running events during low-intensity recovery after ramp-type protocol. Journal of Strength and Conditioning Research, 29(4):10571063. http://dx.doi.org/10.1519/jsc.0000000000000725

Walsh, N. P., Gleeson, M., Shephard, R. J., Gleeson, M., Woods, J. A., Bishop, N. C., Fleshner, M., Green, C., Pedersen, B. K., Hoffman-Goetz, L., Rogers, C. J., Northoff, H., Abbasi, A., \& Simon, P. (2011). Position statement. Part one: Immune function and exercise. Exercise Immunology Reviews, 17, 6-63.

Wirtz, N., Wahl, P., Kleinöder, H., Mester, J. (2014). Lactate Kinetics during Multiple Set Resistance Exercise. Journal of Sports Science and Medicine, 13(1), 73-77. 